\title{
Asymptotic equivalence relations for rapidly varying solutions of sublinear differential equations of Emden-Fowler type
}

\author{
Jelena Manojlović ${ }^{1}$ and Jelena Milošević ${ }^{*}$ (B)
}

"Correspondence:

jefimija79@gmail.com

'Department of Mathematics,

Faculty of Science and

Mathematics, University of Niš,

Višegradska 33, 18000 Niš, Serbia

\begin{abstract}
We discuss sublinear differential equations of the Emden-Fowler type $x^{\prime \prime}=q(t) x^{\gamma}$ under the assumption that the coefficient $q$ is a rapidly varying function. We show that all of their strongly decreasing and strongly increasing solutions are rapidly varying functions and are in the asymptotic equivalence relation with a precisely defined function determined by the coefficient $q$.
\end{abstract}

MSC: Primary 34A34; secondary 26A12

Keywords: Emden-Fowler differential equations; Rapid variation; Strongly decreasing solutions; Strongly increasing solutions

\section{Introduction}

This paper is concerned with positive solutions of differential equations of the EmdenFowler type of the form

$$
x^{\prime \prime}(t)=q(t) x(t)^{\gamma}, \quad t \geq a>0,
$$

where $\gamma \neq 1$ is a positive constant, and $q$ is positive, continuous function on $[a, \infty)$.

Equation (E) is called sublinear or superlinear according to $\gamma<1$ or $\gamma>1$. We consider the sublinear case, i.e., when $0<\gamma<1$.

Any positive solution $x$ of (E), continuable at infinity and eventually different from zero, is either increasing or decreasing. A positive decreasing solution of $(E)$ is said to be

- strongly decreasing if $\lim _{t \rightarrow \infty} x(t)=0, \lim _{t \rightarrow \infty} x^{\prime}(t)=0$,

- asymptotically constant if $\lim _{t \rightarrow \infty} x(t)=$ const $>0, \lim _{t \rightarrow \infty} x^{\prime}(t)=0$.

A positive increasing solution of $(E)$ is said to be

- asymptotically linear if $\lim _{t \rightarrow \infty} x(t)=\infty, \lim _{t \rightarrow \infty} \frac{x(t)}{t}=$ const $>0$,

- strongly increasing if $\lim _{t \rightarrow \infty} x(t)=\infty, \lim _{t \rightarrow \infty} x^{\prime}(t)=\infty$.

The existence of the above four types has been studied in [2, 25]. In the sublinear case, the existence of strongly increasing solutions is completely characterized, while for the existence of strongly decreasing solutions, only the sufficient condition is known, as it is stated in the following propositions.

(c) The Author(s) 2022. This article is licensed under a Creative Commons Attribution 4.0 International License, which permits use sharing, adaptation, distribution and reproduction in any medium or format, as long as you give appropriate credit to the original author(s) and the source, provide a link to the Creative Commons licence, and indicate if changes were made. The images or other third party material in this article are included in the article's Creative Commons licence, unless indicated otherwise in a credit line to the material. If material is not included in the article's Creative Commons licence and your intended use is not permitted by statutory regulation or exceeds the permitted use, you will need to obtain permission directly from the copyright holder. To view a copy of this licence, visit http://creativecommons.org/licenses/by/4.0/. 
Proposition 1.1 ([25, Theorem 3.8]) Sublinear equation (E) has a strongly increasing solution if and only if

$$
\int_{a}^{\infty} t^{\gamma} q(t) d t=\infty
$$

Proposition 1.2 ([25, Theorem 3.2]) Sublinear equation (E) has a strongly decreasing solution if

$$
\int_{a}^{\infty} t q(t) d t<\infty
$$

The existence and asymptotic behavior of regularly varying solutions of nonlinear differential equations were extensively studied in $[8,9,11,13-16,18-22,24]$. Unlike regularly varying solutions, rapidly varying solutions of linear and nonlinear equations are much less studied. The study of second-order linear differential equation in the framework of rapid variation was initiated by Marić [23]. Half-linear differential equations in the framework of the Karamata theory and the de Haan theory were treated in [26-28]. Also, the existence of regularly and rapidly varying solutions of third-order nonlinear differential equations was studied in [17], while in [10,12] the conditions for the existence and asymptotic representations of solutions are given assuming that the coefficient of the equation belongs to the subclass of rapidly varying functions. Although the results in $[10,12]$ can be applied to (E), the problem of determining the conditions for all solutions to be rapidly varying functions is not considered in these papers. Therefore, our goal in this paper is to prove that all strongly decreasing and strongly increasing solutions are rapidly varying functions under the assumption that the coefficient $q$ is rapidly varying and to examine the properties of these solutions in more detail. In addition, the existence conditions and asymptotic representations of solutions are given in $[10,12]$ under the assumption that the coefficient of the equation belongs to the subclass of rapidly varying functions. The solutions considered in these papers also belong to the subclass of rapidly varying functions. Therefore, the results obtained in this paper improve the results in [10,12], since we consider the equation with rapidly varying coefficient and its rapidly varying solutions.

This paper is organized as follows: In Sect. 2, we give the basic definitions and properties of the regularly and rapidly varying functions. We also present asymptotic equivalence relations in the class of rapidly varying functions of index $\infty$, which are defined in $[1,5]$, and some of their basic properties that are useful for our research. In addition, we introduce analogous relations in the class of rapidly varying functions of index $-\infty$ and examine their properties. The main results are stated in Sect. 3. In Sect. 4, we prove some important lemmas that significantly shorten the proof of the main results. Section 5 contains the proofs of the main results. Some illustrative examples are presented in Sect. 6.

\section{Preliminaries}

In this section, first, we recall basic information on the Karamata theory of regularly varying functions and the de Haan theory of rapidly varying functions. 
Definition 2.1 A measurable function $f:[a, \infty) \rightarrow(0, \infty)$ is said to be regularly varying of index $\rho \in \mathbb{R}$ if

$$
\lim _{t \rightarrow \infty} \frac{f(\lambda t)}{f(t)}=\lambda^{\rho} \quad \text { for all } \lambda>0
$$

The set of all regularly varying functions of index $\rho$ is denoted by $\operatorname{RV}(\rho)$.

Definition 2.2 A measurable function $f:[a, \infty) \rightarrow(0, \infty)$ is said to be rapidly varying of index $\infty$ if

$$
\lim _{t \rightarrow \infty} \frac{f(\lambda t)}{f(t)}=\infty \quad \text { for all } \lambda>1 .
$$

A measurable function $f:[a, \infty) \rightarrow(0, \infty)$ is said to be rapidly varying of index $-\infty$ if

$$
\lim _{t \rightarrow \infty} \frac{f(\lambda t)}{f(t)}=0 \quad \text { for all } \lambda>1
$$

The set of rapidly varying functions of index $\infty$ (or $-\infty$ ) is denoted by $\operatorname{RPV}(\infty)$ (or $\mathrm{RPV}(-\infty)$ ). For more information on regular and rapid variation, the reader is referred to the monograph by Bingaham, Goldie, and Teugels [1]. For more recent contribution of the theory of rapid variation, see $[6,7]$.

\section{Example 2.1}

1. It is easy to see that function $f(t)=a^{t}, a>1$ is a typical representative of the class $\operatorname{RPV}(\infty)$, while the function $f(t)=a^{t}, a \in(0,1)$ is a typical representative of the class $\operatorname{RPV}(-\infty)$.

2. The function $f(t)=g(t) a^{h(t)}, g \in \mathrm{RV}(\rho), \rho \in \mathbb{R}, h \in \mathrm{RV}(m), m>0$ belongs to the $\operatorname{RPV}(\infty)$, when $a>1$ and $\operatorname{RPV}(-\infty)$, when $a \in(0,1)$.

Next, we give some properties of rapidly varying functions.

\section{Proposition 2.1}

(1) $f \in \operatorname{RPV}(\infty)$ if and only if $1 / f \in \operatorname{RPV}(-\infty)$.

(2) Iff, $g \in \mathrm{RPV}(\infty)$ and $h \in \mathrm{RV}(\rho), \rho \in \mathbb{R}$, then

(i) $f^{p} \in \mathrm{RPV}(\infty)$ for any $p>0$.

(ii) $f \cdot h \in \mathrm{RPV}(\infty)$.

(iii) $f \cdot g \in \mathrm{RPV}(\infty)$

Proof (1) This part of the proposition is shown in [29] on time scales.

(2) Since $\lim _{t \rightarrow \infty} \frac{f(\lambda t)}{f(t)}=\infty, \lim _{t \rightarrow \infty} \frac{g(\lambda t)}{g(t)}=\infty$ and $\lim _{t \rightarrow \infty} \frac{h(\lambda t)}{h(t)}=\lambda^{\rho}$ for all $\lambda>1$, we have

(i) $\lim _{t \rightarrow \infty} \frac{(f(\lambda t))^{p}}{(f(t))^{p}}=\left(\lim _{t \rightarrow \infty} \frac{f(\lambda t)}{f(t)}\right)^{p}=\infty$, for any $p>0$,

(ii) $\lim _{t \rightarrow \infty} \frac{f(\lambda t) \cdot h(\lambda t)}{f(t) \cdot h(t)}=\lambda^{\rho} \cdot \lim _{t \rightarrow \infty} \frac{f(\lambda t)}{f(t)}=\infty$

(iii) $\lim _{t \rightarrow \infty} \frac{f(\lambda t) \cdot g(\lambda t)}{f(t) \cdot g(t)}=\infty$.

Next, we consider some useful equivalence relations on the classes $\operatorname{RPV}(\infty)$ and $\operatorname{RPV}(-\infty)$. The following relation is introduced in [1] and further considered in $[3,4]$. 
Definition 2.3 Let $f$ and $g$ be positive functions in $[a, \infty)$. These two functions are called mutually inversely asymptotic at $\infty$, denoted by $f(t) \stackrel{\star}{\sim} g(t), t \rightarrow \infty$, if for every $\lambda>1$, there exists $t_{0}=t_{0}(\lambda)$ such that

$$
f\left(\frac{t}{\lambda}\right) \leq g(t) \leq f(\lambda t), \quad \text { for all } t \geq t_{0}
$$

Definition of the next relation and its properties are given in [5].

Definition 2.4 Let $f$ and $g$ be positive functions in $[a, \infty)$. These two functions are called mutually rapidly equivalent at $\infty$, denoted by $f(t) \stackrel{r}{\sim} g(t), t \rightarrow \infty$, if

$$
\lim _{t \rightarrow \infty} \frac{f(\lambda t)}{g(t)}=\lim _{t \rightarrow \infty} \frac{g(\lambda t)}{f(t)}=\infty, \quad \text { for all } \lambda>1 .
$$

Proposition 2.2 Let $f$ and $g$ be positive functions in $[a, \infty)$. Then, the following assertions hold:

(a) if $f$ and $g$ are measurable functions such that $f(t) \stackrel{r}{\sim} g(t)$ for $t \rightarrow \infty$, then $f$ and $g$ both belong to $\mathrm{RPV}(\infty)$;

(b) the relation $\stackrel{r}{\sim}$ is an equivalence relation in the class $\mathrm{RPV}(\infty)$.

Proposition 2.3 Let $f \in \operatorname{RPV}(\infty)$ be a locally bounded function on $[a, \infty)$. Also, let $1 / f$ be a locally bounded function on $[a, \infty)$. Then the following assertions are true:

(a) $f(t) \stackrel{r}{\sim} \frac{1}{t} \int_{a}^{t} f(s) d s, t \rightarrow \infty$;

(b) $f(t) \stackrel{r}{\sim} \frac{1}{t \int_{t}^{\infty} \frac{d s}{s^{2} f(s)}}, t \rightarrow \infty$;

(c) $F \in \operatorname{RPV}(\infty)$, where $F(t)=\int_{a}^{t} f(s) d s, t>a$;

(d) $\varphi \in \operatorname{RPV}(\infty)$, where $\varphi(t)=\frac{1}{\int_{t}^{\infty} \frac{d s}{f(s)}}, t>a$.

Remark 2.1 It is easy to prove that if $f(t) \stackrel{r}{\sim} g(t), t \rightarrow \infty$, then

(a) $f(t)^{p} \stackrel{r}{\sim} g(t)^{p}, t \rightarrow \infty$ for all $p>0$,

(b) $h(t) \cdot f(t) \stackrel{r}{\sim} h(t) \cdot g(t), t \rightarrow \infty$ for $h \in \operatorname{RV}(\rho), \rho \in \mathbb{R}$ or $h \in \operatorname{RPV}(\infty)$.

Here, we introduce two new relations on $\operatorname{RPV}(-\infty)$.

Definition 2.5 Let $f$ and $g$ be positive functions in $[a, \infty)$. These two functions are called mutually inversely asymptotic at $-\infty$, denoted by $f(t) \underset{\star}{\sim} g(t), t \rightarrow \infty$, if for every $\lambda>1$, there exists $t_{0}=t_{0}(\lambda)$ such that

$$
f(\lambda t) \leq g(t) \leq f\left(\frac{t}{\lambda}\right), \quad \text { for all } t \geq t_{0}
$$

Definition 2.6 Let $f$ and $g$ be positive functions in $[a, \infty)$. These two functions are called mutually rapidly equivalent at $-\infty$, denoted by $f(t) \underset{r}{\sim} g(t), t \rightarrow \infty$, if

$$
\lim _{t \rightarrow \infty} \frac{f(\lambda t)}{g(t)}=\lim _{t \rightarrow \infty} \frac{g(\lambda t)}{f(t)}=0, \quad \text { for all } \lambda>1
$$

The next proposition establishes a connection between relations $\stackrel{r}{\sim}$ and $\underset{r}{\sim}$. 
Proposition 2.4 Let $f$ and $g$ be positive functions in $[a, \infty)$. Then

$$
f(t) \stackrel{r}{\sim} g(t), \quad t \rightarrow \infty \quad \text { if and only if } \quad \frac{1}{f(t)} \sim \frac{1}{r} \frac{1}{g(t)}, \quad t \rightarrow \infty .
$$

Proof The proposition directly follows from the equalities

$$
\lim _{t \rightarrow \infty} \frac{f(\lambda t)}{g(t)}=\left[\lim _{t \rightarrow \infty} \frac{\frac{1}{f(\lambda t)}}{\frac{1}{g(t)}}\right]^{-1} \text { and } \lim _{t \rightarrow \infty} \frac{g(\lambda t)}{f(t)}=\left[\lim _{t \rightarrow \infty} \frac{\frac{1}{g(\lambda t)}}{\frac{1}{f(t)}}\right]^{-1} .
$$

The next proposition directly follows from Proposition 2.4, Proposition 2.2, and Proposition 2.1.

Proposition 2.5 Let $f$ and $g$ be positive functions in $[a, \infty)$. Then the following assertions hold:

(a) iff and $g$ are measurable functions such that $f(t) \underset{r}{\sim} g(t)$ for $t \rightarrow \infty$, then $f$ and $g$ both belong to $\mathrm{RPV}(-\infty)$;

(b) the relation $\underset{r}{\sim}$ is an equivalence relation in the class $\mathrm{RPV}(-\infty)$.

Remark 2.2 Proposition 2.3(b) will be easier to use if we rewrite it in a different form. Denote $g(t)=\frac{1}{t^{2} f(t)}$. Hence, due to Remark 2.1, we have

$$
t^{2} f(t) \stackrel{r}{\sim} \frac{t}{\int_{t}^{\infty} g(s) d s}, \quad t \rightarrow \infty
$$

yielding

$$
\frac{1}{t^{2} f(t)} \sim \frac{1}{r} \int_{t}^{\infty} g(s) d s, \quad t \rightarrow \infty
$$

by using the Proposition 2.4. Since $f \in \operatorname{RPV}(\infty)$, from Proposition 2.1, we conclude that $g \in \operatorname{RPV}(-\infty)$. Also, since $1 / f$ is a locally bounded function on $[a, \infty)$, so is $g$.

Therefore, we have the following proposition.

Proposition 2.6 Let $g \in \mathrm{RPV}(-\infty)$ be a locally bounded function on $[a, \infty)$. Then

$$
g(t) \underset{r}{\sim} \frac{1}{t} \int_{t}^{\infty} g(s) d s, \quad t \rightarrow \infty .
$$

\section{Main results}

Theorem 3.1 Suppose that $q \in \mathrm{RPV}(\infty)$ satisfies the condition (1.1). Every strongly increasing solution of $(\mathrm{E})$ is rapidly varying of index $\infty$. Moreover, any such solution $x$ satisfies the asymptotic relation

$$
x(t) \stackrel{\star}{\sim} X(t), \quad t \rightarrow \infty,
$$

where the function $X$ is given by

$$
X(t)=\left(t^{2} q(t)\right)^{\frac{1}{1-\gamma}} .
$$


Theorem 3.2 Suppose that $q \in \mathrm{RPV}(-\infty)$ satisfies the condition (1.2). Every strongly decreasing solution of $(\mathrm{E})$ is rapidly varying of index $-\infty$. Moreover, any such solution $x$ satisfies the asymptotic relation

$$
x(t) \underset{\star}{\sim} X(t), \quad t \rightarrow \infty
$$

\section{Auxiliary lemmas}

Let us denote

$$
\begin{aligned}
& X_{1}(t)=\left(t \int_{a}^{t} q(s) d s\right)^{\frac{1}{1-\gamma}}, \\
& X_{2}(t)=\left(\int_{a}^{t}\left(\int_{a}^{s} q(r)^{\frac{2}{3+\gamma}} d r\right)^{\frac{3+\gamma}{1-\gamma}} d s\right)^{\frac{1}{2}} .
\end{aligned}
$$

First, we show that functions $X, X_{1}$, and $X_{2}$ are in the relation $\stackrel{r}{\sim}$ under the assumption that $q$ is a rapidly varying function of index $\infty$.

Lemma 4.1 Suppose that $q \in \operatorname{RPV}(\infty)$. Then

$$
X(t) \stackrel{r}{\sim} X_{1}(t), \quad t \rightarrow \infty
$$

where the functions $X$ and $X_{1}$ are given by (3.2) and (4.1), respectively.

Proof Using Proposition 2.3(a), we have

$$
q(t) \stackrel{r}{\sim} \frac{1}{t} \int_{a}^{t} q(s) d s, \quad t \rightarrow \infty
$$

Multiplying (4.4) by $t^{2}$, in the view of Remark 2.1, we get

$$
t^{2} q(t) \stackrel{r}{\sim} t \int_{a}^{t} q(s) d s, \quad t \rightarrow \infty
$$

implying

$$
\left(t^{2} q(t)\right)^{\frac{1}{1-\gamma}} \stackrel{r}{\sim}\left(t \int_{a}^{t} q(s) d s\right)^{\frac{1}{1-\gamma}}, \quad t \rightarrow \infty
$$

since $\frac{1}{1-\gamma}>0$. This completes the proof of Lemma 4.1.

Lemma 4.2 Suppose that $q \in \operatorname{RPV}(\infty)$. Then

$$
X(t) \stackrel{r}{\sim} X_{2}(t), \quad t \rightarrow \infty
$$

where the functions $X$ and $X_{2}$ are given by (3.2) and (4.2), respectively. 
Proof Applying Proposition 2.3(a), we conclude that

$$
\int_{a}^{t} q(s)^{\frac{2}{3+\gamma}} d s \stackrel{r}{\sim} t \cdot q(t)^{\frac{2}{3+\gamma}}, \quad t \rightarrow \infty
$$

Since $\frac{3+\gamma}{1-\gamma}>0$, due to Remark 2.1, we get

$$
\left(\int_{a}^{t} q(s)^{\frac{2}{3+\gamma}} d s\right)^{\frac{3+\gamma}{1-\gamma}} \stackrel{r}{\sim} t^{\frac{3+\gamma}{1-\gamma}} q(t)^{\frac{2}{1-\gamma}}, \quad t \rightarrow \infty
$$

On the other hand, another application of Proposition 2.3(a) gives us

$$
\int_{a}^{t}\left(\int_{a}^{s} q(r)^{\frac{2}{3+\gamma}} d r\right)^{\frac{3+\gamma}{1-\gamma}} d s \stackrel{r}{\sim} t\left(\int_{a}^{t} q(s)^{\frac{2}{3+\gamma}} d s\right)^{\frac{3+\gamma}{1-\gamma}}, \quad t \rightarrow \infty
$$

By combining (4.6) and (4.7), we have

$$
\int_{a}^{t}\left(\int_{a}^{s} q(r)^{\frac{2}{3+\gamma}} d r\right)^{\frac{3+\gamma}{1-\gamma}} d s \stackrel{r}{\sim} t^{\frac{4}{1-\gamma}} q(t)^{\frac{2}{1-\gamma}}, \quad t \rightarrow \infty
$$

implying

$$
\left(\int_{a}^{t}\left(\int_{a}^{s} q(r)^{\frac{2}{3+\gamma}} d r\right)^{\frac{3+\gamma}{1-\gamma}} d s\right)^{\frac{1}{2}} \stackrel{r}{\sim}\left(t^{2} q(t)\right)^{\frac{1}{1-\gamma}}, \quad t \rightarrow \infty
$$

This completes the proof of Lemma 4.2.

Denote by

$$
\begin{aligned}
& Y_{1}(t)=\left(\int_{t}^{\infty} \int_{s}^{\infty} q(r) d r d s\right)^{\frac{1}{1-\gamma}} \\
& Y_{2}(t)=\left(\int_{t}^{\infty}\left(\int_{s}^{\infty} q(r)^{\frac{2}{3+\gamma}} d r\right)^{\frac{3+\gamma}{1-\gamma}} d s\right)^{\frac{1}{2}} .
\end{aligned}
$$

Next, we show that functions $X, Y_{1}$, and $Y_{2}$ are in the relation $\underset{r}{\sim}$ under the assumption that $q$ is a rapidly varying function of index $-\infty$.

Lemma 4.3 Suppose that $q \in \operatorname{RPV}(-\infty)$. Then

$$
X(t) \underset{r}{\sim} Y_{1}(t), \quad t \rightarrow \infty
$$

where the functions $X$ and $Y_{1}$ are given by (3.2) and (4.8), respectively.

Proof Using Proposition 2.6, we get

$$
\int_{t}^{\infty} q(s) d s \underset{r}{\sim} t q(t), \quad t \rightarrow \infty
$$


On the other hand, another application of Proposition 2.6 gives us

$$
\int_{t}^{\infty} \int_{s}^{\infty} q(r) d r d s \underset{r}{\sim} t \int_{t}^{\infty} q(s) d s, \quad t \rightarrow \infty
$$

From (4.11) and (4.12), we conclude

$$
\int_{t}^{\infty} \int_{s}^{\infty} q(r) d r d s \underset{r}{\sim} t^{2} q(t), \quad t \rightarrow \infty
$$

implying (4.10).

Lemma 4.4 Suppose that $q \in \operatorname{RPV}(-\infty)$. Then

$$
X(t) \underset{r}{\sim} Y_{2}(t), \quad t \rightarrow \infty
$$

where the functions $X$ and $Y_{2}$ are given by (3.2) and (4.9), respectively.

Proof Applying Proposition 2.6, we conclude that

$$
\int_{t}^{\infty} q(s)^{\frac{2}{3+\gamma}} d s \underset{r}{\sim} t \cdot q(t)^{\frac{2}{3+\gamma}}, \quad t \rightarrow \infty
$$

implying

$$
\left(\int_{t}^{\infty} q(s)^{\frac{2}{3+\gamma}} d s\right)^{\frac{3+\gamma}{1-\gamma}} \underset{r}{\sim} t^{\frac{3+\gamma}{1-\gamma}} q(t)^{\frac{2}{1-\gamma}}, \quad t \rightarrow \infty,
$$

since $\frac{3+\gamma}{1-\gamma}>0$. On the other hand, another use of Proposition 2.6 gives us

$$
\int_{t}^{\infty}\left(\int_{s}^{\infty} q(r)^{\frac{2}{3+\gamma}} d r\right)^{\frac{3+\gamma}{1-\gamma}} d s \underset{r}{\sim} t\left(\int_{t}^{\infty} q(s)^{\frac{2}{3+\gamma}} d s\right)^{\frac{3+\gamma}{1-\gamma}}, \quad t \rightarrow \infty .
$$

By combining (4.14) and (4.15), we get

$$
\int_{t}^{\infty}\left(\int_{s}^{\infty} q(r)^{\frac{2}{3+\gamma}} d r\right)^{\frac{3+\gamma}{1-\gamma}} d s \underset{r}{\sim} t^{\frac{4}{1-\gamma}} q(t)^{\frac{2}{1-\gamma}}, \quad t \rightarrow \infty
$$

yielding (4.13).

\section{Proofs of main results}

Proof of Theorem 3.1 Since $q$ satisfies the condition (1.1), we obtain that the equation (E) has a strongly increasing solution.

Let $x$ be arbitrary strongly increasing solution of (E) defined on $[T, \infty), T \geq a$. First, we show that there exist positive constants $m, M$ such that

$$
m X_{2}(t) \leq x(t) \leq M X_{1}(t), \quad \text { for large } t
$$


where $X_{1}$ and $X_{2}$ are given by (4.1) and (4.2), respectively. Integrating $x^{\prime}$ on $[T, t]$, we get

$$
x(t)=x(T)+\int_{T}^{t} x^{\prime}(s) d s \leq x(T)+x^{\prime}(t)(t-T), \quad t \geq T,
$$

because $x^{\prime}$ is increasing. Hence, we find $K_{1}>0$ such that

$$
x(t) \leq K_{1} t x^{\prime}(t), \quad t \geq T
$$

Since $x$ is increasing, integration of (E) from $T$ to $t$ gives

$$
x^{\prime}(t)=x^{\prime}(T)+\int_{T}^{t} q(s) x(s)^{\gamma} d s \leq x^{\prime}(T)+x(t)^{\gamma} \int_{T}^{t} q(s) d s, \quad t \geq T,
$$

implying, due to the fact $\int_{T}^{t} q(s) d s \rightarrow \infty$ as $t \rightarrow \infty$, that we find $K_{2}>0$ such that

$$
x^{\prime}(t) \leq K_{2} x(t)^{\gamma} \int_{T}^{t} q(s) d s, \quad t \geq T .
$$

By combining (5.2) and (5.3), we have

$$
x(t) \leq K_{1} K_{2} t x(t)^{\gamma} \int_{T}^{t} q(s) d s, \quad t \geq T .
$$

Thus, there exists $M>0$ such that

$$
x(t) \leq M\left(t \int_{T}^{t} q(s) d s\right)^{\frac{1}{1-\gamma}}, \quad t \geq T .
$$

The right-hand side of the inequality (5.1) is proved.

Next, we prove the left-hand side of the inequality (5.1). Set $w(t)=x(t) x^{\prime}(t)$ and

$$
\nu=\frac{\gamma+1}{\gamma+3}, \quad \mu=\frac{2}{\gamma+3}, \quad \kappa=\frac{1-\gamma}{\gamma+3} .
$$

An application of Young's inequality gives

$$
\begin{aligned}
w^{\prime}(t) & =w(t)\left(\frac{q(t) x(t)^{\gamma}}{x^{\prime}(t)}+\frac{x^{\prime}(t)}{x(t)}\right) \geq \frac{w(t)}{\mu^{\mu} \nu^{\nu}}\left(\frac{q(t) x(t)^{\gamma}}{x^{\prime}(t)}\right)^{\mu}\left(\frac{x^{\prime}(t)}{x(t)}\right)^{v} \\
& =\frac{w(t)}{\mu^{\mu} v^{\nu}} x(t)^{\gamma \mu-\nu} x^{\prime}(t)^{\nu-\mu} q(t)^{\mu} .
\end{aligned}
$$

Since, $\gamma \mu-v=v-\mu=-\kappa$, we get

$$
w^{\prime}(t) \geq \frac{1}{\mu^{\mu} \nu^{\nu}} w(t)^{1-\kappa} q(t)^{\mu} .
$$

After dividing (5.5) by $w(t)^{1-\kappa}$ and integrating the obtained inequality on $[T, t]$, we get that there is $k_{1}>0$ such that

$$
w(t)^{\kappa} \geq k_{1} \int_{T}^{t} q(s)^{\mu} d s, \quad t \geq T
$$


or

$$
x(t) x^{\prime}(t) \geq k_{1}^{\frac{1}{\kappa}}\left(\int_{T}^{t} q(s)^{\mu} d s\right)^{\frac{1}{\kappa}}, \quad t \geq T .
$$

Integrating (5.6) from $T$ to $t$, we find $k_{2}>0$ and $T^{*} \geq T$ sufficiently large such that

$$
\frac{x(t)^{2}}{2} \geq k_{2} \int_{a}^{t}\left(\int_{a}^{s} q(r)^{\frac{2}{\gamma+3}} d r\right)^{\frac{\gamma+3}{1-\gamma}} d s, \quad t \geq T^{*}
$$

From (5.7), we obtain that there exists $m>0$ such that the left-hand side of the inequality (5.1) is satisfied.

Next, we prove that $x$ is a rapidly varying function of index $\infty$. Fix arbitrary $\lambda>1$. Indeed, from (5.1) for sufficiently large $t$, we have

$$
m X_{2}(\lambda t) \leq x(\lambda t) \leq M X_{1}(\lambda t)
$$

and

$$
\frac{1}{M X_{1}(t)} \leq \frac{1}{x(t)} \leq \frac{1}{m X_{2}(t)}
$$

From (5.8) and (5.9), we obtain

$$
\frac{m}{M} \frac{X_{2}(\lambda t)}{X_{1}(t)} \leq \frac{x(\lambda t)}{x(t)} \leq \frac{M}{m} \frac{X_{1}(\lambda t)}{X_{2}(t)}
$$

for sufficiently large $t$. By Lemma 4.1 and Lemma 4.2 , we have $X_{1}(t) \stackrel{r}{\sim} X_{2}(t), t \rightarrow \infty$, which means

$$
\lim _{t \rightarrow \infty} \frac{X_{2}(\lambda t)}{X_{1}(t)}=\lim _{t \rightarrow \infty} \frac{X_{1}(\lambda t)}{X_{2}(t)}=\infty .
$$

Since $\lambda$ was arbitrary, combining (5.10) and (5.11) gives us $\lim _{t \rightarrow \infty} \frac{x(\lambda t)}{x(t)}=\infty$ for all $\lambda>1$, that is, $x \in \operatorname{RPV}(\infty)$.

It remains to prove that the solution $x$ satisfies the asymptotic relation (3.1). Fix arbitrary $\lambda>1$. Let $m$ and $M$ be positive numbers, satisfying (5.1) for $t \geq T_{1} \geq T$. By Lemma 4.1 and Lemma 4.2, we have (4.3) and (4.5), so there exists $T_{2}=T_{2}(\lambda) \geq T_{1}$ such that

$$
M X_{1}(t) \leq X(\lambda t) \wedge X\left(\frac{t}{\lambda}\right) \leq m X_{2}(t), \quad t \geq T_{2} .
$$

Therefore, from (5.1), we conclude that

$$
X\left(\frac{t}{\lambda}\right) \leq x(t) \leq X(\lambda t), \quad t \geq T_{2}
$$

implying $x(t) \stackrel{\star}{\sim} X(t), t \rightarrow \infty$. This completes the proof of Theorem 3.1. 
Proof of Theorem 3.2 Assumption (1.2) ensures the existence of strongly decreasing solution of $(E)$. Assume that $x$ is the arbitrary strongly decreasing solution of (E) defined on $[T, \infty), T \geq a$. First, we show that there exist positive constants $m$ and $M$ such that

$$
m Y_{2}(t) \leq x(t) \leq M Y_{1}(t), \quad \text { for large } t
$$

where $Y_{1}$ and $Y_{2}$ are given by (4.8) and (4.9), respectively. Since $x^{\prime}(t) \rightarrow 0, t \rightarrow \infty$, and $x$ is decreasing, integrating $(\mathrm{E})$ from $t$ to $\infty$, we get

$$
-x^{\prime}(t)=\int_{t}^{\infty} q(s) x(s)^{\gamma} d s \leq x(t)^{\gamma} \int_{t}^{\infty} q(s) d s, \quad t \geq T .
$$

Dividing (5.14) by $x(t)^{\gamma}$ and then integrating from $t$ to $\infty$, since $x(t) \rightarrow 0, t \rightarrow \infty$, we have

$$
\frac{1}{1-\gamma} x(t)^{1-\gamma} \leq \int_{t}^{\infty} \int_{s}^{\infty} q(r) d r d s, \quad t \geq T
$$

implying that there exists $M>0$ such that the right-hand side of the inequality (5.13) is satisfied.

Next, we prove the left-hand side of the inequality (5.13). Setting $w(t)=x(t)\left|x^{\prime}(t)\right|$ and $v$, $\mu, \kappa$ as in (5.4), application of Young's inequality gives

$$
\begin{aligned}
-w^{\prime}(t) & =w(t)\left(\frac{q(t) x(t)^{\gamma}}{\left|x^{\prime}(t)\right|}+\frac{\left|x^{\prime}(t)\right|}{x(t)}\right) \geq \frac{w(t)}{\mu^{\mu} v^{\nu}}\left(\frac{q(t) x(t)^{\gamma}}{\left|x^{\prime}(t)\right|}\right)^{\mu}\left(\frac{\left|x^{\prime}(t)\right|}{x(t)}\right)^{\nu} \\
& =\frac{w(t)}{\mu^{\mu} \nu^{\nu}} x(t)^{\gamma \mu-\nu}\left|x^{\prime}(t)\right|^{\nu-\mu} q(t)^{\mu}=\frac{1}{\mu^{\mu} v^{\nu}} w(t)^{1-\kappa} q(t)^{\mu},
\end{aligned}
$$

afterwards multiplying by $w(t)^{\kappa-1}$ and integrating from $t$ to $\infty$, we find $k_{1}>0$ such that

$$
w(t)^{\kappa} \geq k_{1} \int_{t}^{\infty} q(s)^{\mu} d s, \quad t \geq T
$$

or

$$
-x(t) x^{\prime}(t) \geq k_{1}^{\frac{1}{\kappa}}\left(\int_{t}^{\infty} q(s)^{\mu} d s\right)^{\frac{1}{\kappa}}, \quad t \geq T .
$$

Since $x(t) \rightarrow 0, t \rightarrow \infty$, integrating (5.15) from $t$ to $\infty$ yields that there is $k_{2}>0$ such that

$$
\frac{x(t)^{2}}{2} \geq k_{2} \int_{t}^{\infty}\left(\int_{s}^{\infty} q(r)^{\frac{2}{\gamma+3}} d r\right)^{\frac{\gamma+3}{1-\gamma}} d s .
$$

From (5.16), we obtain that there exists $m>0$ such that the left-hand side of the inequality (5.13) is satisfied.

That $x \in \mathrm{RPV}(-\infty)$ and satisfies the asymptotic relation (3.3) can be proved in the same way as in the proof of Theorem 3.1, using Lemma 4.3 and Lemma 4.4. 


\section{Examples}

Now, we present two examples that illustrate main results stated by Theorem 3.1 and Theorem 3.2.

Example 6.1 Consider the equation

$$
x^{\prime \prime}(t)=q_{1}(t) x^{\gamma}(t), \quad t>0,0<\gamma<1,
$$

where $q_{1}(t)=e^{t+(1-\gamma) e^{t}}\left(1+e^{t}\right)$. Since

$$
q_{1} \in \mathrm{RPV}(\infty) \wedge \int_{a}^{\infty} t^{\gamma} q_{1}(t) d t=\infty
$$

by Theorem 3.1 follows that every strongly increasing solution of (6.1) is rapidly varying of index $\infty$, and any such solution $x$ satisfies the asymptotic relation

$$
x(t) \stackrel{\star}{\sim} Q_{1}(t), \quad t \rightarrow \infty
$$

where $Q_{1}(t)=\left(t^{2} q_{1}(t)\right)^{\frac{1}{1-\gamma}}$. It is easy to check that $x_{1}(t)=e^{e^{t}}$ is such a solution of (6.1), since $x_{1} \in \operatorname{RPV}(\infty)$ and

$$
\lim _{t \rightarrow \infty} \frac{x_{1}(\lambda t)}{Q_{1}(t)}=\lim _{t \rightarrow \infty} \frac{Q_{1}(\lambda t)}{x_{1}(t)}=\infty,
$$

implying that $x_{1}$ satisfies the asymptotic relation (6.2).

Example 6.2 Consider the equation

$$
x^{\prime \prime}(t)=q_{2}(t) x^{\gamma}(t), \quad t>0,0<\gamma<1,
$$

where $q_{2}(t)=k^{2} e^{k(\gamma-1) t}, k>0$. Since

$$
q_{2} \in \operatorname{RPV}(-\infty) \wedge \int_{a}^{\infty} t q_{2}(t) d t<\infty
$$

by Theorem 3.2 follows that every strongly decreasing solution of (6.3) is rapidly varying of index $-\infty$, and any such solution $x$ satisfies the asymptotic relation

$$
x(t) \underset{\star}{\sim} Q_{2}(t), \quad t \rightarrow \infty,
$$

where $Q_{2}(t)=\left(t^{2} q_{2}(t)\right)^{\frac{1}{1-\gamma}}$. It is easy to check that $x_{2}(t)=e^{-k t}, k>0$ is such a solution of (6.3), since $x_{2} \in \operatorname{RPV}(-\infty)$ and

$$
\lim _{t \rightarrow \infty} \frac{x_{2}(\lambda t)}{Q_{2}(t)}=\lim _{t \rightarrow \infty} \frac{Q_{2}(\lambda t)}{x_{2}(t)}=0,
$$

implying that $x_{2}$ satisfies the asymptotic relation (6.4). 


\section{Acknowledgements}

Not applicable.

\section{Funding}

Corresponding author Jelena Milošević is not currently in receipt of any research funding. Author Jelena Manojlović acknowledges financial support through the Ministry of Education and Science of Republic of Serbia, agreement no. 451-03-68/2022-14/200124.

\section{Availability of data and materials}

Not applicable.

\section{Declarations}

\section{Competing interests}

The authors declare that they have no competing interests.

\section{Authors' contributions}

Both authors have equally contributed to the writing of this paper. Moreover, all authors have read and approved the manuscript.

\section{Publisher's Note}

Springer Nature remains neutral with regard to jurisdictional claims in published maps and institutional affiliations.

\section{Received: 23 April 2021 Accepted: 4 February 2022 Published online: 03 March 2022}

\section{References}

1. Bingham, N.H., Goldie, C.M., Teugels, J.L.: Regular Variation. Encyclopedia of Mathematics and Its Applications, vol. 27. Cambridge University Press, Cambridge (1987)

2. Cecchi, M., Došlá, Z., Marini, M.: Integral conditions for nonoscillation of second order nonlinear differential equations. Nonlinear Anal. 64, 1278-1289 (2006)

3. Djurčić, D., Mitrović, I., Janjić, M.: The weak and the strong equivalence relation and the asymptotic inversion. Filomat 25(4), 29-36 (2011)

4. Djurčić, D., Torgašev, A., Ješić, S.: The strong asymptotic equivalence and the generalized inverse. Sib. Math. J. 49, 628-636 (2008)

5. Elez, N., Djurčić, D.: Some properties of rapidly varying functions. J. Math. Anal. Appl. 401, 888-895 (2013)

6. Elez, N., Djurčić, D.: Rapid variability and Karamata's integral theorem. Filomat 28(3), 487-492 (2014)

7. Elez, N., Djurčić, D.: Representation and characterization of rapidly varying functions. Hacet. J. Math. Stat. 44(2), 317-322 (2015)

8. Evtukhov, V.M., Doroshenko, A.G.: Asymptotic behavior of slotuions of ordinary differential equationa of the $n$-th order with regularly varying nonlinearities. J. Math. Sci. 243(2), 204-229 (2019)

9. Evtukhov, V.M., Droggina, A.V.: Asymptotic representations for the solutions of nonautonomous ordinary differential equations. Ukr. Math. J. 71(12), 1865-1887 (2020)

10. Evtukhov, V.M., Drozhzhina, A.V.: Asymptotics of rapidly varying solutions of differential equations asymptotically close to equations with regularly varaying nonlinearities. J. Math. Sci. 253(2), 242-262 (2021)

11. Evtukhov, V.M., Klopot, A.M.: Asymptotic behavior of solutions of $n$ th-order ordinary differential equations with regularly varying nonlinearities. Differ. Equ. 50(5), 1-17 (2014)

12. Evtukhov, V.M., Kolun, N.: Rapidly varying solutions of a second-order differential equation with regularly and rapidly varying nonlinearities. J. Math. Sci. 235(1), 15-34 (2018)

13. Evtukhov, V.M., Kolun, N.: Asymptotic behavior of solutions of second-order nonlinear diferential equations. Mem. Differ. Equ. Math. Phys. 75, 105-114 (2018)

14. Evtukhov, V.M., Kolun, N.: Asymptotic representations of the solutions of differential equations with regularly and rapidly varying nonlinearities. J. Math. Sci. 240(1), 34-47 (2019)

15. Jaroš, J., Kusano, T.: Slowly varying solutions of a class of first order systems of nonlinear differential equations. Acta Math. Univ. Comen. 82, 265-284 (2013)

16. Jaroš, J., Kusano, T.: Existence and precise asymptotic behavior of strongly monotone solutions of systems of nonlinear differential equations. Differ. Equ. Appl. 5, 185-204 (2013)

17. Jaroš, J., Kusano, T., Marić, V.: Existence of regularly and rapidly varying solutions for a class of third order nonlinear ordinary differential equations. Publ. Inst. Math. 79(93), 51-64 (2006)

18. Kočinac, L., Djurčić, D., Manojlović, J.: Regular and rapid variations and some applications. In: Ruzhansky, M., Dutta, H., Agarwal, R.P. (eds.) Mathematical Analysis and Applications: Selected Topics, pp. 429-491. Wiley, New York (2018)

19. Kusano, T., Manojlović, J., Marić, V.: Increasing solutions of Thomas-Fermi type differential equations-the sublinear case. Bull. Cl. Sci. Math. Nat. Sci. Math. 36, 21-36 (2011)

20. Kusano, T., Manojlović, J., Marić, V.: Increasing solutions of Thomas-Fermi type differential equations-the superlinear case. Nonlinear Anal., Theory Methods Appl. 108, 114-127 (2014)

21. Kusano, T., Manojlović, J., Marić, V.: Complete asymptotic analysis of second-order differential equations of Thomas-Fermi type in the framework of regular variation. Sūrikaisekikenkyūsho Kōkyūroku, No. 1959, 2015.7, Qualitative theory of ordinary differential equations in real domains and its applications, pp. 14-34 (2015). http://www.kurims.kyoto-u.ac.jp/ kyodo/kokyuroku/contents/1959.html

22. Manojlović, J., Marić, V:. An asymptotic analysis of positive solutions of Thomas-Fermi type sublinear differential equations. Mem. Differ. Equ. Math. Phys. 57, 75-94 (2012) 
23. Marić, V.: Regular Variation and Differential Equations. Lecture Notes in Mathematics, vol. 1726. Springer, Berlin (2000)

24. Matucci, S., Rehák, P.: Asymptotics of decreasing solutions of coupled p-Laplacian systems in the framework of regular variation. Ann. Mat. Pura Appl. 193(3), 837-858 (2014)

25. Mizukami, M., Naito, M., Usami, H.: Asymptotic behavior of solutions of a class of second order quasilinear ordinary differential equations. Hiroshima Math. J. 32(1), 51-78 (2002)

26. R̆ehák, P.: De Haan type increasing solutions of half-linear differential equations. J. Math. Anal. Appl. 412, 236-243 (2014)

27. Rehák, P.: Half-linear differential equations, the Karamata theory, and the de Haan theory. Res. Inst. Math. Sci., Kyoto Univ. 1993, 1-25 (2016). http://hdl.handle.net/2433/224658

28. Rehák, P., Taddei, V.: Solutions of half-linear differential equations in the classes Gamma and Pi. Differ. Integral Equ. 29(7/8), 683-714 (2016)

29. Vítovec, J.: Theory of rapid variation on time scales with applications to dynamic equations. Arch. Math. 46(4), 263-284 (2010)

\section{Submit your manuscript to a SpringerOpen ${ }^{\circ}$} journal and benefit from:

- Convenient online submission

- Rigorous peer review

- Open access: articles freely available online

- High visibility within the field

- Retaining the copyright to your article

Submit your next manuscript at $\gg$ springeropen.com 